\title{
Wie kann die Dokumentation in der Physiotherapie durch die Digitalisierung effektiver und effizienter werden?
}

\author{
Eine Delphi-Studie
}

\section{How Can Documentation in Physiotherapy Be Made More Effective and Efficient through Digitization?}

\author{
A Delphi Study
}

Autoren

Annika Griefahn' ${ }^{1}$ Eduard Wolf², Christoff Zalpour ${ }^{1}$

Institute

1 Hochschule Osnabrück, Fakultät Wirtschafts- und Sozialwissenschaften, Osnabrück, Deutschland

2 Hochschule Osnabrück, Fakultät Ingenieurwissenschaften und Informatik, Osnabrück, Deutschland

Schlüsselwörter

Delphi-Studie, Dokumentation, Physiotherapie, Software

Key words

delphi study, documentation, physiotherapy, software

eingereicht 11.09.2019

akzeptiert 08.01.2020

Bibliografie

DOI https://doi.org/10.1055/a-1113-6688

Online-Publikation: 28.4.2020

physioscience 2020; 16: 101-110

(c) Georg Thieme Verlag KG, Stuttgart · New York

ISSN 1860-3092

Korrespondenzadresse

Annika Griefahn, PT, BSc PT, MSc Sportphysiotherapie

Hochschule Osnabrück, Albrechtstraße 30, 49076 Osnabrück

a.griefahn@hs-osnabrueck.de

\section{ZUSAMMENFASSUNG}

Hintergrund Die physiotherapeutische Dokumentation spielt im Therapieprozess eine wichtige Rolle, erfolgt jedoch auf unterschiedlichste Weise. Es existieren derzeit eine Vielzahl von Softwarelösungen für die physiotherapeutische Dokumentation, welche sich jedoch in ihren Funktionalitäten stark unterscheiden.

Ziel Mithilfe einer Befragung soll ein Konsens von Experten aus der Physiotherapie im Themengebiet der softwarebasierten Dokumentation ermittelt werden. Anhand der Ergebnisse wird ein Anforderungskatalog für die Entwicklung einer neuartigen und benutzerorientierten Dokumentationssoftware erstellt.

Methode Online-Delphi-Befragung mit neun Experten über drei Befragungswellen.
Ergebnisse Hinsichtlich der Anforderungen an die Anamnese konnte ein Konsens erzielt werden. Bei der Gliederung des Befundes kam es zu keiner Übereinstimmung der Experten. Das Ergebnis lässt sich durch unterschiedliche manualtherapeutische Konzepte erklären, die für die Befunderhebung verwendet wurden.

Schlussfolgerung Eine softwarebasierte Dokumentation sollte standardisierter als bisher erfolgen, um den ClinicalReasoning-Prozess zu unterstützten. Gleichzeitig ist dabei eine gewisse Flexibilität geboten. Die gesammelten Anforderungen können für die Entwicklung einer neuartigen und benutzerorientierten mobilen Anwendung zur Effizienzsteigerung in der physiotherapeutischen Dokumentation verwendet werden.

\section{ABSTRACT}

Background Physiotherapeutic documentation is an important factor in the therapy process, however it is done in different ways. There are currently a large number of software solutions for physiotherapeutic documentation, which differ greatly in their functionalities.

Objective A survey was conducted to find a consensus of physiotherapists in the area of software-based documentation. Based on these results, a catalog of requirements for the development of a novel and user-oriented documentation software was to be created.

Method An online-delphi-survey with nine experts over three survey waves was conducted.

Results Consensus could be achieved with regard to the requirements for the anamnesis. In the structure of the findings no agreement was reached between the experts. This can be explained by different manual therapeutic concepts, which were used to structure the findings.

Conclusion The survey indicates that software-based documentation should be standardized to support the clinical reasoning process, while providing some flexibility at the same time. The collected requirements are to be used for the development of a novel and user-oriented mobile application for increasing the efficiency in physiotherapeutic documentation. 


\section{Einleitung}

Die Dokumentation eines Therapieverlaufes gehört zum klinischen Alltag von Physiotherapeuten. Die physiotherapeutische Dokumentation umfasst mehrere Teilbereiche. Im Bürgerlichen Gesetzbuch (BGB) §630 f. ist festgelegt, dass Heilmittelerbringer folgende Punkte dokumentieren müssen:

- Durchführung der Befunderhebung

- Aufstellen eines individuellen Behandlungsplans

- Durchführung der therapeutischen Maßnahmen

- Regelbehandlungszeit

- Verlaufsdokumentation

- ggf. Mitteilung an den verordneten Arzt [1]

Das Gesetz verpflichtet die Heilmittelerbringer auch dazu, die erbrachten Leistungen (z. B. Manuelle Therapie) auf dem neusten Stand wissenschaftlicher Erkenntnisse durchgeführt zu haben. Darüber hinaus soll die Sicherung der Ergebnisqualität (vgl. Sozialgesetzbuch (SGB), Fünftes Buch (V), §135a, Verpflichtung der Leistungserbringer zur Qualitätssicherung) erfolgen, indem die erbrachten Leistungen stets weiterentwickelt werden [2]. Eine weitere rechtliche Anforderung stellt das Patientenrechtegesetz im BGB dar, das im Februar 2013 in Kraft trat. Dieses Gesetz umfasst die Regelung der Behandlungsverträge, die Aufklärung und Informationsweitergabe zwischen Physiotherapeuten und Patienten sowie die Dokumentationspflicht, falls es zu Rechtsstreitigkeiten kommt [3].

Für die Dokumentation und Erstellung von Abschlussberichten gibt es bis dato keine separate Vergütung, sie werden nicht als Abrechnungsposten im Heilmittelkatalog aufgeführt. Das bedeutet, dass die Dokumentation während der Behandlungszeit eines Patienten erfolgen muss, um die Wirtschaftlichkeit zu gewährleisten $[4,5]$.

Neben den gesetzlichen Vorgaben unterstützt die Dokumentation Physiotherapeuten im Clinical-Reasoning-Prozess [6]. Mit Clinical Reasoning werden die Entscheidungs- und Denkprozesse von Physiotherapeuten während der Befundaufnahme und der Therapie bezeichnet. Clinical Reasoning trägt zum reflektierten Handeln im Therapiealltag bei. Eine transparente und nachvollziehbare Dokumentation ist dafür eine wesentliche Grundlage. Aufgrund einer mangelhaften oder fehlenden Dokumentation kann das Handeln während eines Therapieprozesses falsch interpretiert werden oder es kann zu Missverständnissen kommen $[6,7]$.

Die Nutzung von computergestützten Hilfsmitteln ist im Gesundheitssektor mittlerweile weit verbreitet. Dabei findet die Digitalisierung nicht nur in den therapeutischen Berufen statt, sondern auch in der Pflege und in Arztpraxen [8]. Die Hilfsmittel werden dabei vor allem für die Terminplanung, Kommunikation und Abrechnung verwendet. Einige Softwareanbieter werben zusätzlich mit digitaler Befundung und Therapiedokumentation im Bereich der Physiotherapie [8]. Die 27 Millionen in Deutschland ausgestellten physiotherapeutischen Verordnungen werden in den meisten Einrichtungen zwar digital organisiert und verwaltet, die Dokumentation findet hingegen oft noch in Papierform statt [9].
Derzeit existiert eine Vielzahl von Softwarelösungen zur physiotherapeutischen Dokumentation, welche sich inhaltlich und optisch stark voneinander unterscheiden. Alle Lösungen bieten eine gewisse Strukturierung der Dokumentation, enthalten jedoch keine Funktionen, die zur Zeitersparnis beitragen, wie z. B. eine Sprachunterstützung oder eine automatische Generierung von Therapieberichten.

\section{Methode}

Zunächst erfolgte eine systematische Literaturrecherche mit vordefinierten Suchbegriffen, welche die Rahmenbedingungen und Anforderungen an die physiotherapeutische Dokumentation aufzeigen. Die Literaturrecherche umfasste für die elektronische Dokumentation folgende Suchbegriffe mit den entsprechenden Synonymen: Anamnese, Befund, digital, Dokumentation, Funktion, Nutzen, Physiotherapie und Software. Die vordefinierten Suchbegriffe für die rechtlichen Grundlagen waren: Physiotherapie, Dokumentation, Gesetz und Datenschutz. Die Suchbegriffe wurden mit den Booleschen Operatoren sinnvoll verknüpft und auf die Datenbanken PubMed, Cochrane, Datenbank des Deutschen Institutes für medizinische Information und Dokumentation (DIMDI) sowie das juris-Informationssystem der Bundesrepublik Deutschland angewendet.

Die Ergebnisse der Literaturrecherche zeigen, dass gesetzliche Vorgaben existieren [1, 2], Rahmenverträge der Krankenkassen als Richtlinien dienen [4] und unterschiedliche Dokumentationsweisen zur Anwendung kommen [3]. Für die Entwicklung einer Dokumentationssoftware sind aber nicht nur die rechtlichen Bestimmungen relevant, sondern auch die Meinungen von Experten, wie eine Erleichterung der Dokumentation und Kommunikation stattfinden kann. Um diese Expertenmeinungen einzuholen, wurde eine Delphi-Befragung mit Physiotherapeuten durchgeführt. Die Manuskriptdarstellung folgte der Leitlinie CREDES (Conducting and REporting DElphi Studies) [10].

\section{Delphi-Befragung}

Das Ziel der Delphi-Befragung war die Sammlung von Anforderungen zur Erstellung eines Anforderungskataloges für die Entwicklung einer physiotherapeutischen Dokumentationssoftware zur Erleichterung der Dokumentation.

Im Gesundheitssektor findet die Delphi-Befragung häufig Anwendung [8, 9] und ist eine spezifische Form der Gruppenkommunikation [13]. Die Delphi-Befragung ermöglicht das systematische Sammeln von Meinungen zu einem bestimmten Themengebiet. Die Meinungen der Experten wurden in der vorliegenden Delphi-Befragung zuerst qualitativ abgefragt und anschließend mit weiteren Fragebögen quantifiziert. Durch die Quantifizierung können die Ergebnisse mittels eines Medians oder Mittelwertes abgebildet bzw. ausgewertet werden [14]. Zu den Besonderheiten einer Delphi-Befragung gehören die wiederkehrende Reflektion nach jeder Befragungswelle sowie die Anonymisierung der Experten [15]. Für die Inhaltsvalidierung der Fragebögen wurden vorab PreTests mithilfe einer Monitoringgruppe durchgeführt. Ziel dieser Pre-Tests war es, missverständliche Fragen zu klären, die Bearbeitungszeit abzuschätzen sowie die zu erwartenden Antworten abzu- 
gleichen [15]. Anschließend erfolgte eine Überarbeitung der Fragebögen. \ Abb. 1 zeigt ein Flussdiagramm zum Delphi-Prozess.

\section{Expertengruppe}

Als Voraussetzung für die Inklusion in die Expertengruppe wurden mindestens fünf Jahre Berufserfahrung in einer leitenden Position vorausgesetzt. Die Panelgröße schwankt in der Literatur und ist bei einer qualitativen Befragung auf maximal zehn Teilnehmer begrenzt [15].

\section{Messinstrument - Fragebogen}

Für die Entwicklung der Fragebögen wurde die Leitfrage „Wie kann die physiotherapeutische Dokumentation erleichtert werden?“ formuliert. Die Leitfrage wurde in die zwei Teilbereiche „Dokumentation“ und „Erleichterung“ operationalisiert. Dadurch konnte ein Fragebogen mit offenen Fragen entwickelt werden, der dazu diente, das Problemfeld zu strukturieren. Die ausschließlich offen formulierten Fragen wurden gewählt, um die Experten nicht in ihrer Meinung zu beeinflussen. Der Fragebogen der ersten Befragungswelle umfasste insgesamt 27 Fragen. Nach Abschluss der ersten Befragungswelle wurden die Antworten systematisch aufgelistet. Es erfolgte eine sprachliche Vereinheitlichung, Doppelungen wurden gestrichen. Die Aufarbeitung der Antworten diente zur Einteilung quantitativer Fragekategorien. Die Antworten aus der ersten Befragungswelle wurden anschließend wieder in Abschnitte aufgeteilt und es erfolgte eine Quantifizierung mittels geschlossener Fragen innerhalb der zweiten Befragungswelle. Dieser Fragebogen bestand aus insgesamt 28 Fragen, die in vier Abschnitte unterteilt wurden. Auf Grundlage der Ergebnisauswertung wurde anschließend die dritte Befragungswelle mit einem finalen Fragebogen mit insgesamt 63 Fragen entwickelt. \ Tab. 1 zeigt die drei Befragungswellen im Überblick.

Die Fragebögen der Delphi-Befragung wurden als Onlineformular mithilfe von Google Forms (www.google.com/forms/) an die Experten übermittelt.

\section{Auswertung}

Für die statistische Auswertung wurden zwei Verfahren angewendet. Die qualitativen Ergebnisse aus der ersten Befragungswelle wurden mit einem qualitativ-deskriptiven Ansatz ausgewertet [15]. Dabei wurden die Freitexte von zwei unterschiedlichen Untersuchern zusammengefasst sowie in Kategorien eingeordnet. Ziel dieser Auswertung war es, Antwortmöglichkeiten für neu entwickelte Fragen zusammenzustellen. Die deskriptive Auswertung der zweiten und dritten Befragungswelle erfolgte mittels Microsoft Excel. Es wurden Mediane und Mittelwerte für die Antworten erfasst und verarbeitet [16].

\section{Ergebnisse}

\section{Expertengruppe}

Die Befragung erfolgte mit insgesamt neun Experten, sie setzte sich aus acht männlichen Teilnehmern und einer weiblichen Teilnehmerin zusammen. In $>$ Tab. 2 sind die soziodemografischen Daten der Experten dargestellt. Die Experten waren entweder

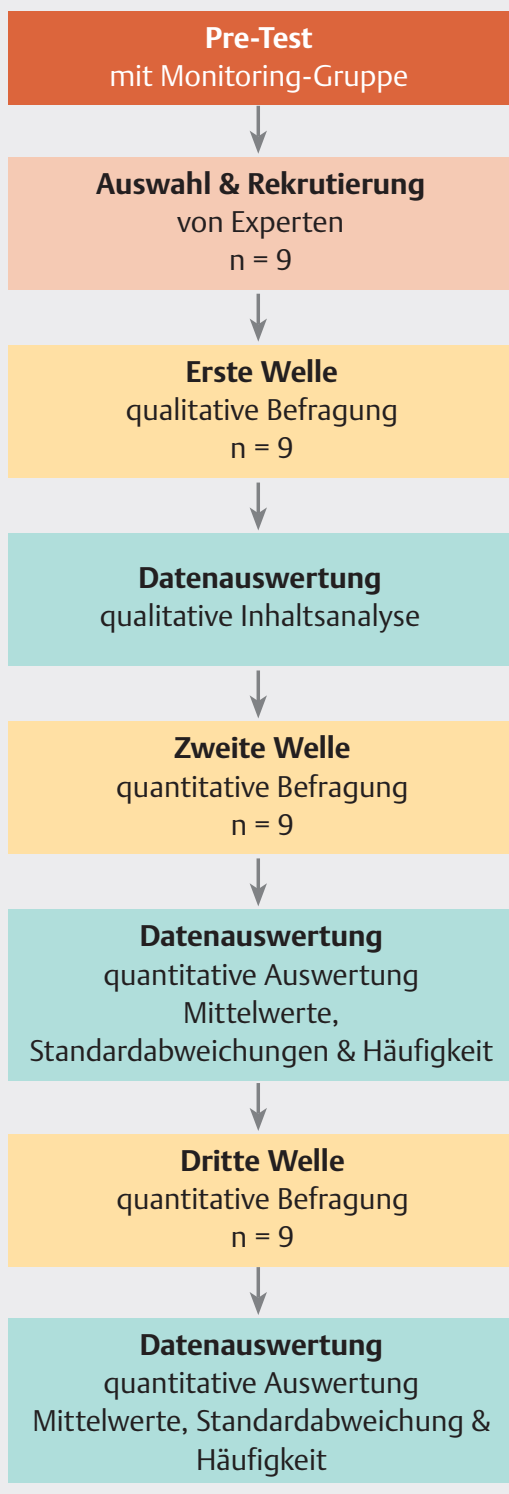

- Abb. 1 Der Delphi-Prozess als Flussdiagramm.

(Quelle: A. Griefahn; graf. Umsetzung: Thieme Gruppe)

selbstständig oder hatten eine leitende Position in einer Physiotherapiepraxis. Die Fortbildungen erstreckten sich von Manuelle Therapie über Orthopädische Manuelle Therapie (IFOMPT-Standard) bis zur Sportphysiotherapie. Die Schwerpunkte lagen in der konservativen Behandlung sowie dem Sportsektor.

Da die Experten aus dem orthopädischen Bereich kamen, beschränkte sich die Delphi-Befragung auf die Inhalte in der orthopädischen Behandlung. Andere Inhalte wie z. B. in der neurologischen oder pädiatrischen Dokumentation fanden keine Berücksichtigung.

\section{Erste Befragungswelle}

Die Ergebnisse der ersten Befragungswelle sind in $\mathbf{A b b} \mathbf{2}$ dargestellt. Nachfolgend werden zunächst die Ergebnisse des ersten Teils des Fragebogens beschrieben. Zuerst wurden die üblichen 
- Tab. 1 Darstellung der drei Befragungswellen mit Thematik und Fragenanzahl.

\section{Erste Befragungswelle}

\begin{tabular}{|l|l|l|}
\hline Abschnitt & Dokumentation & 15 Fragen \\
\hline Abschnitt & Dokumentationserleichterung & 6 Fragen \\
\hline Abschnitt & soziodemografische Fragen & 6 Fragen \\
\hline
\end{tabular}

Zweite Befragungswelle

\begin{tabular}{|l|l|l|}
\hline Abschnitt & Dokumentationsinhalte & 17 Fragen \\
\hline Abschnitt & Dokumentationsweise & 1 Frage \\
\hline Abschnitt & Dokumentationserleichterung & 6 Fragen \\
\hline Abschnitt & $\begin{array}{l}\text { Anschaffung einer Dokumentations- } \\
\text { software }\end{array}$ & 4 Fragen \\
\hline
\end{tabular}

Dritte Befragungswelle

\begin{tabular}{|c|l|l|}
\hline Abschnitt & Dokumentationsweise & 6 Fragen \\
\hline Abschnitt & Patientenakte & 3 Fragen \\
\hline Abschnitt & Anamnese und Befund & 16 Fragen \\
\hline Abschnitt & Verlaufsdokumentation & 5 Fragen \\
\hline Abschnitt & Therapiebericht & 5 Fragen \\
\hline Abschnitt & Sprachunterstützung & 10 Fragen \\
\hline Abschnitt & Maßnahmen und Assessments & 7 Fragen \\
\hline Abschnitt & Fragebögen und Formulare & 3 Fragen \\
\hline Abschnitt & technische Anforderungen & 7 Fragen \\
\hline
\end{tabular}

- Tab. 2 Soziodemografische Daten der Experten.

\begin{tabular}{|c|c|}
\hline Expertengruppe & $\begin{array}{l}8 \times \text { männlich } \\
1 \times \text { weiblich }\end{array}$ \\
\hline Durchschnittsalter & 45,38 Jahre $(S D \pm 10,24)$ \\
\hline Berufserfahrung & 23 Jahre $(S D \pm 9,93)$ \\
\hline $\begin{array}{l}\text { höchster Bildungsab- } \\
\text { schluss }\end{array}$ & $\begin{array}{l}6 \times \text { Hochschulreife } \\
2 \times \text { Fachhochschulreife } \\
1 \times \text { sonstiger Bildungsabschluss }\end{array}$ \\
\hline $\begin{array}{l}\text { höchster beruflicher } \\
\text { Bildungsabschluss }\end{array}$ & $\begin{array}{l}5 \times \text { Master-Abschluss } \\
2 \times \text { Bachelor-Abschluss } \\
2 \times \text { Staatlich geprüfter Physiothera- } \\
\text { peut/Staatlich geprüfte Physiothera- } \\
\text { peutin }\end{array}$ \\
\hline
\end{tabular}

Dokumentationsinhalte abgefragt. Die Punkte Anamnese, Behandlungsdaten und Ziele wurden dabei am häufigsten genannt. Zusätzliche Nennungen waren: Untersuchung, Patientendaten, Maßnahmen, Schmerzhistorie und Befund.

Zur Frage, in welcher Reihenfolge die genannten Inhalte dokumentiert werden, lässt sich feststellen, dass zu Beginn eine Anlage des Patienten mit Rezeptprüfung erfolgt. Dann werden Anamnese, Befund und Zielvereinbarung dokumentiert. Im letzten Schritt erfolgt die Dokumentation des Therapieverlaufs sowie der durch- geführten Maßnahmen. Die Ergebnisse zur Frage, welche Aspekte der Dokumentation aufgrund von Zeitmangel oder weil sie impraktikabel sind, häufig vernachlässigt werden, zeigen, dass es vor allem bei Dauerpatienten zu einer Vernachlässigung der Dokumentation kommt. Auch der Therapieverlauf und Abschlussbefund werden häufig nicht dokumentiert. Ziele, Re-Test-Verfahren und Hausaufgaben werden aufgrund von Zeitmangel ebenfalls nicht dokumentiert.

Hinsichtlich der Dokumentation der Anamnese wurden als Schwerpunkte die Identifikation der Beschwerden, Vorerkrankungen, vorherige Behandlungen sowie Medikation genannt. Wie sich bei der Befragung zeigte, werden im Befund mehrere Parameter dokumentiert. Diese Parameter setzen sich aus unterschiedlichen Testverfahren wie z. B. Mobilitäts-, Kraft- oder Safety-Tests zusammen. Bei diesen Testungen werden jedoch nur positive Untersuchungsergebnisse notiert. Neben den diversen Testungen werden das Hauptproblem und die Diagnose dokumentiert. Ebenso wird von einigen Teilnehmenden der Sicht- und Tastbefund unter Verwendung einer Körpertabelle erfasst. Im Therapieverlauf werden die Veränderungen der Testparameter und der Patient selbst sowie die durchgeführte Intervention erfasst. Die Ergebnisse bezüglich der gewünschten Funktionalität einer Dokumentationssoftware zeigen, dass vor allem Vorlagen bzw. Leitfäden sowie eine Sammlung von Assessments gefordert werden. Von einigen Experten wurde zudem die Nutzung einer Spracheingabe sowie eines Aufklappmenüs angeführt.

Als (mögliche) Vorteile einer softwaregestützten Dokumentation wurden Zeitersparnis, Standardisierung und Gedächtnisstützen genannt. Als mögliche Nachteile wurden erhöhte Kosten und eine geringe Flexibilität aufgeführt. Die Hälfte der befragten Experten verwendet bereits eine Software für die Patientenverwaltung und Abrechnung. Doch nur zwei Experten nutzen eine Software zur Dokumentation. Über die dafür verwendete Software führten sie jedoch nur wenige positive Aspekte an. Dazu zählen die Verwendung einer Körpertabelle sowie eine zentrale Datenhaltung. Zu den negativen Aspekten zählen: oberflächliche Dokumentation, veraltete Benutzeroberfläche, fehlende Kopplung mit mobilen Endgeräten sowie fehlende Strukturierung des Therapieprozesses. Experten, die keine Software zur Dokumentation nutzen, dokumentieren entweder auf Karteikarten, auf formfreiem Papier oder in Microsoft Word.

Im zweiten Teil der ersten Befragungswelle wurden Indikatoren für eine Erleichterung der physiotherapeutischen Dokumentation ermittelt. Es wurde zunächst nach Möglichkeiten gefragt, wie die Dokumentation im Allgemeinen erleichtert bzw. vereinfacht werden kann. Dabei zeigte sich, dass die Nutzung von mobilen Endgeräten sowie eine intuitive und übersichtliche Bedienung die größten Erleichterungen beinhalten würden. Zusätzlich wurden Sprachsteuerung, Möglichkeiten zur Zeitersparnis und Transparenz genannt.

Zur Frage, welche Aspekte durch eine softwaregestützte Dokumentation erleichtert werden, gaben die Experten an, diese erleichtere die Kommunikation zwischen den unterschiedlichen Berufszweigen, ermögliche eine Zeitersparnis sowie eine bessere Standardisierung. Die Ergebnisse der Frage, welche Softwarefunktionen eine Dokumentation erleichtern, zeigen, dass das Spei- 


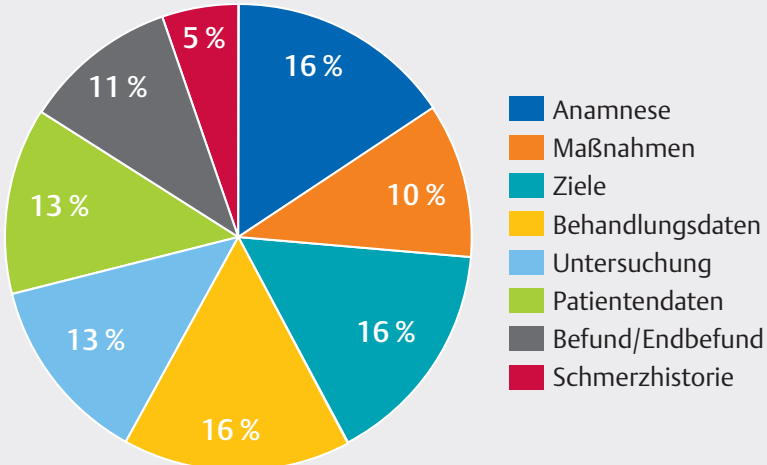

\section{Möglichkeiten für eine softwaregestützte Dokumentation}
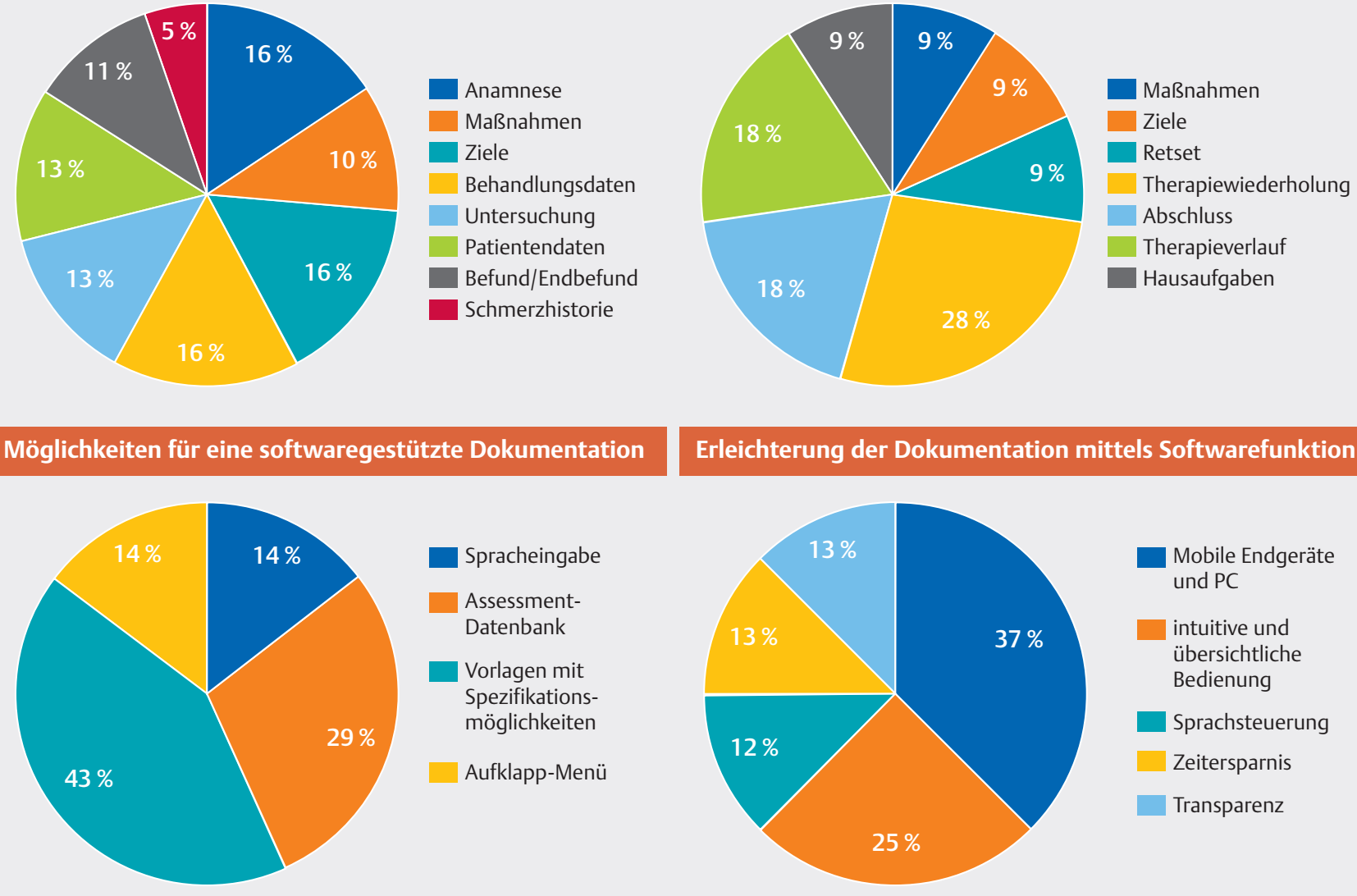

Spracheingabe

AssessmentDatenbank

Vorlagen mit Spezifikationsmöglichkeiten

Aufklapp-Menü

\section{Erleichterung der Dokumentation mittels Softwarefunktion}

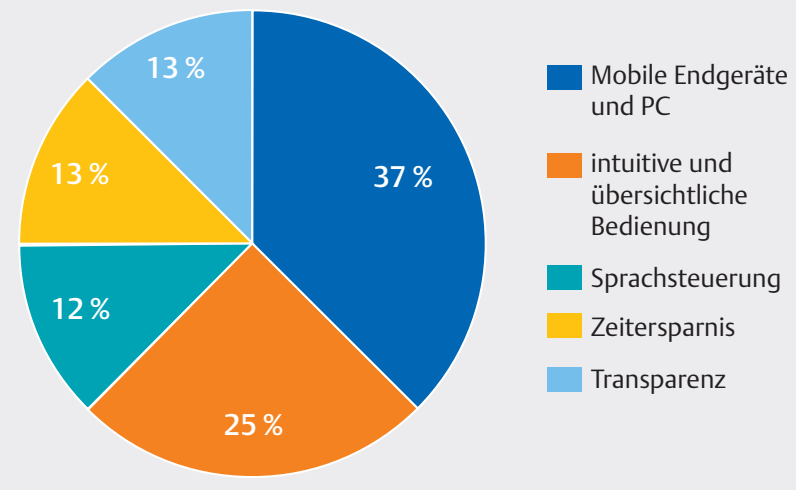

- Abb.2 Die Ergebnisse der ersten Befragungswelle. (Quelle: A. Griefahn; graf. Umsetzung: Thieme Gruppe)

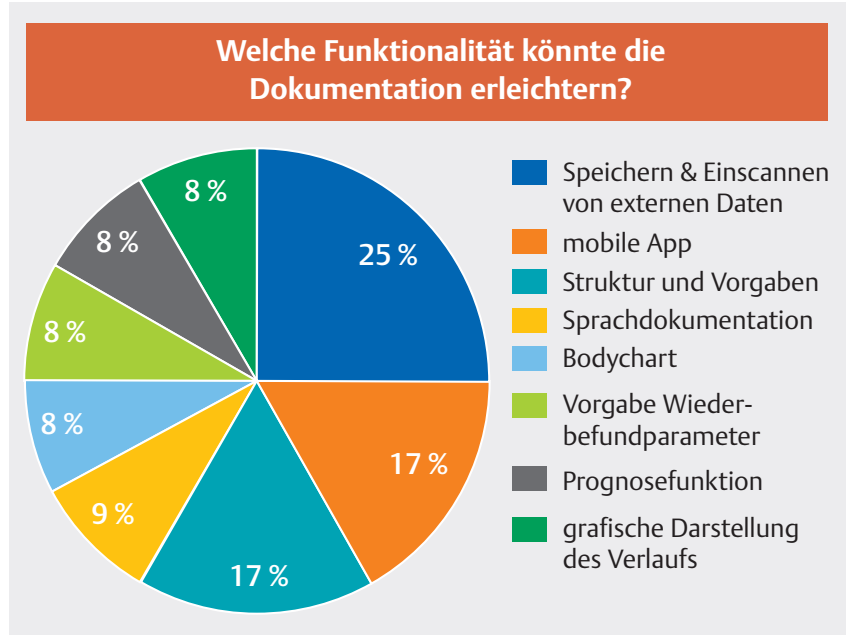

- Abb. 3 Die Ergebnisse zur Funktionalität einer softwaregestützten Dokumentation. (Quelle: A. Griefahn; graf. Umsetzung: Thieme Gruppe)

chern und Scannen externer Dokumente, die Nutzung auf mobilen Endgeräten sowie die Strukturierung der Dokumentation wichtig sind.
Wie in $>$ Abb. 3 zu sehen, wurden die Funktionalitäten Spracheingabe, Körpertabellen, Vorgabe von Wiederbefundparametern, Prognosefunktion sowie grafische Verlaufsdarstellung bei der Frage nach einer Erleichterung durch eine softwaregestützte Dokumentation genannt.

Bei der abschließenden Frage zeigte sich, dass die befragten Experten sich wünschen, dass Patienten selbstständig ihren Beruf, Adresse, Geburtsdatum sowie Hobbies dokumentieren können. Weitere Experten fordern zudem das selbständige Ausfüllen von standardisierten Fragebögen durch die Patienten.

\section{Zweite Befragungswelle}

Im Rahmen der zweiten Befragungswelle stellte sich heraus, dass in den entsprechenden Physiotherapiepraxen der befragten Experten sechsmal das Maitland-Konzept und viermal das Kaltenborn-Konzept zur Befundaufnahme eingesetzt wurden. Eine Mehrfachnennung war möglich. Im ersten Abschnitt der Befragung wurde die Dokumentationsweise abgefragt. In $>$ Tab. 3 sind die allgemeinen Inhalte sowie die Inhalte während der Anamnese mit deren Priorität dargestellt.

Weiterhin wurden der zeitliche Umfang und der Zeitpunkt der einzelnen Dokumentationsschritte abgefragt. Die Dokumentation der Anamnese dauert zwischen 5 und 15 Minuten und findet während der Anamnese statt. Die Befunddokumentation dauert zwi- 
- Tab. 3 Die Prioritäten der Dokumentationsinhalte.

\begin{tabular}{|l|l|l|}
\hline Priorität & Allgemein & Anamnese \\
\hline sehr wichtig & - Anamnese & - Auslöser \\
& - Befund & - Nebenerkrankungen \\
& - Therapiemaßnahmen & - Hauptproblem \\
\hline wichtiger & - Therapieziele & - Schmerzqualität \\
\hline & - Behandlungsverlauf/ & - Historie \\
\hline vorgehen & - Ursachen \\
\hline wichtig & - Ergebnisse & - Vorerkrankungen \\
\hline weniger & - Assessments & - aktuelle Medikation \\
\hline wichtig & - Patientenziele & - Alter \\
\hline unwichtig & - kritische Selbstreflektion & - fabbiliärer Kontext \\
\hline & - Flags & - bisherige Therapien \\
& - Kommentare von & - 24-Stunden- \\
& Patienten & \\
\hline & - Heimprogramme & \\
\hline
\end{tabular}

schen 5 und 10 Minuten und wird ebenfalls während des Befundes durchgeführt. Die Dokumentation der Intervention erfolgt stichpunktartig während der Intervention und der anschließenden Nachbereitung. In der Regel dauert die Dokumentation fünf Minuten. Der Abschlussbericht nimmt 5 bis 10 Minuten Zeit in Anspruch und wird nach der Therapiesitzung dokumentiert.

Im zweiten Abschnitt wurden die regelmäßig genutzten Assessments abgefragt. Dabei zeigte sich, dass fünf Experten die visuelle Analogskala (VAS) verwenden, drei Experten das Goniometer und zwei Experten das Algometer. Darüber hinaus wurde nach einer Möglichkeit zur Unterscheidung von Erst- und Abschlussbefund gefragt. Anhand der Antworten wird ersichtlich, dass sechs Experten eine Unterscheidungsmöglichkeit fordern. Im nächsten Abschnitt wurden Fragen zum Therapieverlauf gestellt. In > Tab. 4 wird deutlich, dass fünf Experten die Dokumentation des Feedbacks als sehr wichtig deklarieren, ebenso die Re-Tests. Als weniger wichtig wird die Dokumentation der exakten Therapie angesehen.

Im dritten Abschnitt wurden Fragen zur Dokumentationserleichterung abgefragt, welche mithilfe von Softwarefunktionalitäten erfolgen können. Textbausteine, eine automatische Berichterstellung, Test- und Assessmentvorschläge sowie eine anatomische Struktur werden als sehr wichtig bewertet. Zudem werden eine Sprachunterstützung sowie Gedächtnisstützen als Erleichterung angegeben. Als hilfreich werden außerdem das selbstständige Ausfüllen des Patientenfragebogens sowie eine digitale Patientenakte erachtet.

Im letzten Abschnitt wurden Fragen zur Auffassung gegenüber der Kostenspanne und der Anschaffung von Endgeräten gestellt. Es zeigte sich, dass fünf Experten eine Dokumentationssoftware neben einer Software zur Terminierung und Abrechnung nutzen würden. Die akzeptable Kostenspanne für eine Anschaffung erstreckt sich von 0 bis 1000 Euro. Bevorzugte Endgeräte sind Tablets oder Smartphones.
D Tab.4 Die Dokumentationsinhalte des Therapieverlaufs.

\begin{tabular}{l|l|l|}
\hline sehr wichtig & wichtig & weniger wichtig \\
\hline - Feedback & " Test & " exakte Therapie \\
\hline - Re-Test & " Hausaufgaben & \\
\hline & Ziele & \\
\hline
\end{tabular}

\section{Dritte Befragungswelle}

In der dritten Befragungswelle lag der Schwerpunkt in der Funktionsweise der Dokumentationssoftware. Die Antworten der Experten machten deutlich, dass eine eher freie bis geleitete Abfrage nach Vorgaben bevorzugt wird. Daneben wünschen sich die befragten Experten eine flexible Konfiguration der Inhaltsstruktur. Eine im Vorfeld von Patienten bestätigte Einverständniserklärung wird als unwichtig, der Import von Gesundheitskartendaten hingegen als sehr wichtig bewertet. Zudem gaben die Experten an, dass eine Erfolgskontrolle der erreichten Therapieziele während der Verlaufsdokumentation hilfreich sei sowie ein Hinweis zu fehlenden Informationen aus Anamnese und Befund. Die Verwendung des manualtherapeutischen Sprachgebrauchs wird als neutral eingestuft. Weiterhin sei die Ausgabe eines Therapieberichts im ICF-Format wichtig sowie die Möglichkeit, eigene Berichtsvorlagen zu erstellen. Eine digitale Übermittlung von Berichten an Ärzte wird als sehr wichtig angesehen. In > Tab. 5 wird die Reihenfolge der einzelnen Dokumentationsschritte bei Anamnese, Schmerz- und Symptomverhalten, Historie und Befund aufgeführt.

Im Abschnitt „Maßnahmen und Assessments“ wurde die Wichtigkeit einer Integration von Clinical Pathways abgefragt, die als weniger wichtig bis wichtig bewertet wird. Als hilfreich werden Assessmentvorschläge sowie das eigenständige Einpflegen von Assessments angesehen. Ebenfalls hilfreich seien standardisierte Fragebögen zur Erfolgskontrolle sowie das Einpflegen digitaler Fragebögen.

Beim Abschnitt „technische Anforderungen“ stellte sich heraus, dass die Experten iOS und Android als mobile Plattformen bevorzugen. Dabei war ihnen eine Kompatibilität mit PC und Mac ebenso wichtig wie eine Offline-Nutzung. Die Verwaltung von mehreren Therapeuten innerhalb der Dokumentationssoftware sowie der freie Wechsel von Endgeräten werden von den Experten als sehr wichtig bewertet. Zusätzlich werden Hinweise und die Dokumentation von Red- und Yellow-Flags als sehr wichtig bewertet. Die Antworten auf die Frage, zu welchem Zeitpunkt eine Körpertabelle verfügbar sein sollte, zeigte, dass es eine Möglichkeit geben sollte, um auf eine Körpertabelle dauerhaft zugreifen zu können. Zur Markierung von Schmerzzonen wünschen sich die Experten die Möglichkeit, diese selbst frei einzeichnen zu können. Weitere Funktionalitäten wie das Markieren positiver Befundergebnisse für den Wiederbefund sowie die Möglichkeit für die Erstellung von Fotos und Videos für den Haltungsbefund werden als sehr hilfreich bewertet. Die Antworten auf die Frage nach einer Sprachunterstützung zeigten, dass eine Spracheingabe bzw. Diktierfunktion als egal bis sehr hilfreich bewertet werden. Eine Sprachsteuerung der Anwendung wird als sehr hilfreich bewertet. 
- Tab.5 Reihenfolge der Dokumentationsschritte bei Anamnese, Schmerz- und Symptomverhalten, Historie und Befund.

\begin{tabular}{|c|c|c|c|}
\hline Anamnese & Schmerz- und Symptomverhalten & Historie & Befund \\
\hline $\begin{array}{l}\text { " allg. Patientendaten } \\
\text { " Hauptproblem } \\
\text { - Schmerz und Symptome } \\
\text { " Historie } \\
\text { " bisherige Therapien } \\
\text { - Vor- und Nebenerkrankungen } \\
\text { " aktuelle Medikation } \\
\text { " familiärer Kontext } \\
\text { - Vorsichtsmaßnahmen } \\
\text { - Patientenziele } \\
\text { - Therapeutenziele }\end{array}$ & $\begin{array}{l}\text { - Lokalisation } \\
\text { - Qualität } \\
\text { - Intensität } \\
\text { - Irritierbarkeit } \\
\text { - Ursache } \\
\text { - Beginn } \\
\text { - Auslöser } \\
\text { - Umstände } \\
\text { - Vermeidungsstrategien }\end{array}$ & $\begin{array}{l}\text { " seit wann } \\
\text { " wie entstanden } \\
\text { " Auslöser } \\
\text { - Verlauf } \\
\text { " Vorgeschichte } \\
\text { - bisherige Therapie }\end{array}$ & $\begin{array}{l}\text { - Statik } \\
\text { - Schonhaltung } \\
\text { - funktionelle Demo } \\
\text { - aktive Bewegung } \\
\text { - passive Bewegung } \\
\text { - Kraftausmaß } \\
\text { - Sicherheitstest } \\
\text { - neurologische Testung } \\
\text { - neurodynamische Testung }\end{array}$ \\
\hline
\end{tabular}

Ein Audiofeedback wird jedoch als störend beurteilt. Außerdem wird eine Lernfunktion für die Erkennung spezieller physiotherapeutischer Begriffe als sehr hilfreich bewertet.

\section{Diskussion}

Die durchgeführte Delphi-Befragung hatte zum Ziel, Anforderungen an eine Dokumentationssoftware für die Physiotherapie zu definieren. Hierfür wurde die Leitfrage „Wie kann die physiotherapeutische Dokumentation erleichtert werden?" formuliert. Diese Leitfrage wurde in zwei Teilbereiche „Dokumentation“ und „Erleichterung“ operationalisiert. Mithilfe der Ergebnisse konnten schließlich Anforderungen an eine Dokumentationssoftware für die Physiotherapie definiert werden.

\section{Ergebnisse}

In Deutschland sind die notwendigen Dokumentationsinhalte im BGB §630f, §630g und V. SGB §135a gesetzlich festgelegt. Seit 2013 gilt außerdem das Patientenrechtegesetz. Dieses Gesetz soll eine verbesserte Gesundheitsversorgung gewährleisten und Patienten bei Behandlungsfehlern stärker unterstützen. Zu den gesetzlich vorgeschriebenen Dokumentationsinhalten zählen Anamnese, Diagnose, Untersuchung, Untersuchungsergebnis, Befund, Therapie und Wirkung sowie Aufklärung, Einwilligung und Therapiebericht. Diese Vorgaben dürfen Patienten einsehen, sie dienen darüber hinaus der Qualitätssicherung.

Bei der durchgeführten Delphi-Befragung lagen die Themenschwerpunkte auf den Dokumentationsinhalten sowie dem Dokumentationsprozess mit dem Ziel, diesen Prozess mithilfe einer softwarebasierten Dokumentation zu optimieren und damit insbesondere Zeit einsparen zu können. Anhand der Ergebnisse wird ersichtlich, dass Dokumentationsinhalte (z. B. Abschlussbericht, Befund, Therapieverlauf und Ziel) häufig vernachlässigt werden, obwohl deren Dokumentation gesetzlich vorgegeben ist. Als Gründe dafür wurden von den Experten Zeitmangel angegeben und dass der Vorgang impraktikabel sei. Der zeitliche Umfang für die Dokumentation beträgt 5 bis 15 Minuten innerhalb der Therapieeinheit, wobei der Therapieverlauf nach der Behandlung in Stichpunkten dokumentiert wird.
Die aktuell verfügbaren Softwarelösungen zur Dokumentation in der Physiotherapie bieten hinsichtlich der gesammelten Anforderungen keine adäquate Lösung. Sie werden häufig nur für Administrationszwecke eingesetzt und nicht für die Dokumentation selbst. Es fehlen oft eine Funktionalität zur umfassenden Dokumentation, die Bedienung ist nicht intuitiv, es entstehen zusätzliche Kosten und es mangelt an der Strukturierung der Therapieprozesse. Deshalb existiert der Bedarf an einer Alternative, welche eine umfangreiche Unterstützung im Therapie- und Dokumentationsprozess bietet.

Neben der Bereitstellung als mobile Anwendung würde die Vorgabe von Wiederbefundparametern, eine Prognosefunktion oder eine Assessmentdatenbank die Dokumentation erleichtern, ebenso wie eine Sprachunterstützung mit Erkennung von physiotherapeutischen Begriffen sowie die grafische Darstellung von Messverläufen (z. B. als VAS oder Bewegungsausmaß). Bei der Analyse von bereits existierenden Dokumentationslösungen und deren Funktionalitäten zeigte sich, dass sich bereits einige Software-Anbieter auf die physiotherapeutische Dokumentation spezialisiert haben. Dabei können weitestgehend die vorgegebenen Dokumentationsinhalte notiert werden, jedoch oft nicht in strukturierter Form. Weitere häufige Funktionen sind Körpertabellen und Foto- bzw. Dokumentenupload. Es fehlt jedoch eine intuitive Benutzeroberfläche (Usability) sowie eine berührungslose Eingabe z. B. mittels Sprachunterstützung.

Eine Dokumentationssoftware mit der gewünschten Funktionalität könnte dazu beitragen, die vorhandene Nachlässigkeit bei der Dokumentation zu verringern und die rechtlichen Rahmenbedingungen einzuhalten. Eine umfassende digitale Dokumentation würde dafür sorgen, dass die Behandlung und der Clinical-Reasoning-Prozess von Physiotherapeuten transparenter wird und die Effizienz während einer Therapieeinheit gesteigert werden kann. Eine softwarebasierte Dokumentation fördert die Digitalisierung in der Physiotherapie und den interdisziplinären Austausch, wodurch der Behandlungserfolg positiv beeinflusst werden kann $[17,18]$. Unter dem Begriff des interdisziplinären Austausches wird in diesem Fall die Kommunikation zwischen unterschiedlichen Gesundheitsberufen verstanden. Zudem kann die Einbindung einer e-Health-Gesundheitsakte sowie die Berücksichtigung von Datenschutzrichtlinien im Rahmen des e-Health-Gesetzes leichter als bisher erfolgen. 


\section{Limitierung}

Im Konzept einer Delphi-Befragung ist festgelegt, dass nur Experten aus dem zugehörigen Themengebiet befragt werden. Es wäre jedoch interessant zu erfahren, welche Dokumentationsinhalte in welchem Umfang Novizen im Vergleich zu Experten leisten. Darüber hinaus bestand die Expertengruppe nur aus Physiotherapeuten, die entweder selbstständig oder in einer leitenden Position tätig sind. Es wäre daher interessant zu erfahren, welche Dokumentationsinhalte in welchem Umfang Physiotherapeuten dokumentieren, die zwar schon lange im Beruf tätig sind, nach den hier definierten Einschlusskriterien aber nicht zu den Experten zählen. Zudem stellte sich heraus, dass die befragte Gruppe sehr homogen war hinsichtlich der Fortbildungen (Manuelle Therapie nach Kaltenborn und/oder Maitland) und Schwerpunkte (orthopädischer konservativer Bereich). Deshalb wäre es interessant, welche Dokumentationsinhalte in welchem Umfang Experten aus anderen Konzepten der Manuellen Therapie oder aus anderen Bereichen wie der neurologischen oder pädiatrischen Therapie dokumentieren.

Das Patientenrechtegesetz regelt die Dokumentation und den Datenschutz. So ist festgelegt, dass die Dokumentation, unabhängig davon, ob sie in Papierform oder digital erstellt wurde, bis zu zehn Jahren aufbewahrt werden muss, und Änderungen nachvollziehbar sein müssen. Es werden jedoch keine genauen Vorgaben zur Speicherung der Daten oder zum Datenschutz gemacht. Es gibt auch keine genaue Beschreibung zur zukünftigen Umsetzung der e-Health-Gesetze, d. h., die Bundesregierung hat noch keine Rahmenbedingungen für die Einführung einer flächendeckenden elektronischen Dokumentation geschaffen, wie es sie z. B. in Finnland gibt.

Die Dokumentationsinhalte sind zwar gesetzlich festgelegt worden, es gibt aber keine Vorschriften zum konkreten Umfang der Inhalte. Eine klare Definition der konkreten inhaltlichen Bestandteile könnte es Therapeuten jedoch ermöglichen, Patientendaten vergleichbar zu machen. Weitere Kritikpunkte sind die Aufnahme und die weiterführende Nutzung von sensiblen Patientendaten. Derweil ist gesetzlich nicht geregelt, inwiefern ein Patient einer elektronischen Dokumentation widersprechen kann, wenn ihm z. B. keine genaue Auskunft über den Speicherort seiner Daten gegeben wird. Zudem besteht die Möglichkeit einer cloudbasierten oder lokalen Speicherung, wobei die zehnjährige Aufbewahrungspflicht berücksichtigt werden muss. Da der Schutz sensibler Patientendaten gegenüber kriminellen Zugriffen stets gewährleistet sein muss, entstehen zusätzliche Kosten für die regelmäßige Wartung und Instandhaltung der Serverinfrastruktur zur Absicherung der Patientendaten.

\section{Anforderungskatalog}

Mit den abgeleiteten Ergebnissen aus der Delphi-Befragung, einem interprofessionellen Team aus Physiotherapeuten und einem Softwareentwickler konnte ein Anforderungskatalog ausgearbeitet werden ( $\triangleright$ Tab. 6). Dieser sollte als Grundlage zur Entwicklung einer neuen Dokumentationssoftware dienen. Der Anforderungskatalog macht deutlich, dass diese Software zum einen die
- Tab. 6 Anforderungskatalog.

\begin{tabular}{|c|c|}
\hline Kategorie & Funktionalität \\
\hline Allgemein & $\begin{array}{l}\text { Benutzerkonto } \\
\text { - Verwalten von Therapeuten } \\
\text { - Jeder Therapeut hat Leserecht aller Patienten- } \\
\text { daten. } \\
\text { - Nur der zum aktuellen Zeitpunkt behandelnde } \\
\text { Therapeut kann vorhandene Dokumentations- } \\
\text { einträge bearbeiten. } \\
\text { - Die Behandlung eines Patienten kann von einem } \\
\text { Therapeuten übernommen werden. } \\
\text { - Jeder Eintrag wird mit Kürzel des Therapeuten } \\
\text { und Zeitstempel versehen. } \\
\text { Geleitete Dokumentation } \\
\text { - Der Therapeut wird je nach Zeitpunkt im } \\
\text { Behandlungsverlauf durch die Dokumentation } \\
\text { geleitet, um den Prozess zu unterstützen. } \\
\text { - Gliederung in } \\
\text { - neue Therapie starten } \\
\text { (Patient } \rightarrow \text { Anamnese } \rightarrow \text { Befund } \rightarrow \text { Intervention), } \\
\text { - Therapie fortsetzen und } \\
\text { (Zusammenfassung } \rightarrow \text { Wiederbefund } \rightarrow \text { Inter- } \\
\text { vention), } \\
\text { - Therapie beenden (Abschlussbefund } \rightarrow \text { Bericht). } \\
\text { - Die Dokumentationsinhalte wie Anamnese, } \\
\text { Befund etc. können auch zu jedem Zeitpunkt für } \\
\text { einen Patienten aufgerufen und bearbeitet } \\
\text { werden. } \\
\text { - Es wird eine Vorkonfiguration der Dokumenta- } \\
\text { tionsinhalte vorgeschlagen, jedoch kann der } \\
\text { Administrator die einzelnen Kategorien anpassen } \\
\text { (z. B. Ursache, Auslöser etc. der Rubrik Schmerz- } \\
\text { verhalten). } \\
\text { - Bei Fortsetzung der Therapie wird auf fehlende } \\
\text { Daten hingewiesen (z. B. Anamnese vervollstän- } \\
\text { digen o. ä.). } \\
\text { Sprachunterstützung } \\
\text { - Transkription von Sprache (Speech-to-Text) mit } \\
\text { Erkennung physiotherapeutischer Fachbegriffe } \\
\text { (Diktierfunktion) } \\
\text { - Lernfunktion der Spracherkennung/benutzerde- } \\
\text { finiertes Wörterbuch } \\
\text { - Ausschalten der Sprachunterstützung } \\
\text { Datenspeicherung } \\
\text { - lokales Speichern der Daten } \\
\text { - Synchronisation der Daten mit einer zentralen } \\
\text { Watel zwischen verschiedenen Geräten }\end{array}$ \\
\hline Patientenakte & $\begin{array}{l}\text { - Verwalten von Patienten } \\
\text { " Vermerken, ob Arztbericht gewünscht ist (Ja/Nein) } \\
\text { Stammdaten } \\
\text { - Eintragen von Namen, Adresse etc. } \\
\text { Dateien und Dokumente } \\
\text { - Hinzufügen von Berichten, Röntgenbildern, } \\
\text { Rezepten etc. als Dokumente }\end{array}$ \\
\hline Körpertabelle & $\begin{array}{l}\text { - Standarddarstellung von jeder Seite } \\
\text { - Zoom oder Auswahl spezifischer Bereiche } \\
\text { - manuelles Einzeichnen mit Farbauswahl } \\
\text { - Hinzufügen von Bemerkungen }\end{array}$ \\
\hline
\end{tabular}


- Tab. 6 (Fortsetzung)

\begin{tabular}{|c|c|}
\hline Kategorie & Funktionalität \\
\hline Anamnese & $\begin{array}{l}\text { Allgemeine Patienteninformationen } \\
\text { Hauptproblem } \\
\text { " Auswahl der Schmerzintensität auf einer visuellen } \\
\text { Analogskala (VAS) } \\
\text { Schmerz-/Symptomverhalten im Tagesverlauf } \\
\text { - Auswahl der Schmerzintensität auf einer visuellen } \\
\text { Analogskala (VAS) } \\
\text { Historie } \\
\text { " freies Eintragen oder Einsprechen } \\
\text { bisherige Therapien } \\
\text { - freies Eintragen oder Einsprechen } \\
\text { Vorerkrankungen } \\
\text { - freies Eintragen oder Einsprechen } \\
\text { Nebenerkrankungen } \\
\text { - freies Eintragen oder Einsprechen } \\
\text { aktuelle Medikation } \\
\text { - freies Eintragen oder Einsprechen } \\
\text { - Unterscheidung von Dauermedikation und } \\
\text { symptombezogener Medikation } \\
\text { familiärer Kontext } \\
\text { - freies Eintragen oder Einsprechen } \\
\text { Vorsichtsmaßnahmen/Kontraindikation } \\
\text { - freies Eintragen oder Einsprechen } \\
\text { Red-Flags/Yellow-Flags } \\
\text { - Auswahl vorgegebener Red-Flags/Yellow-Flags } \\
\text { - Hinweise für eine weitere medizinische Abspra- } \\
\text { che auf Basis positiver Flags } \\
\text { Patientenziele } \\
\text { - freies Eintragen oder Einsprechen } \\
\text { Therapeutenziele } \\
\text { - freies Eintragen oder Einsprechen } \\
\text { Hypothesen } \\
\text { - freies Eintragen oder Einsprechen von Hypothesen }\end{array}$ \\
\hline Befund & $\begin{array}{l}\text { - Darstellung einer Körpertabelle } \\
\text { - keine Festlegung auf ein manualtherapeutisches } \\
\text { Konzept (möglichst allgemeingültig) } \\
\text { - Wird in Erst-, Wieder- und Abschlussbefund } \\
\text { unterteilt. } \\
\text { - Markierung positiver Befundergebnisse für den } \\
\text { Wiederbefund } \\
\text { Haltungsbefund/Statik } \\
\text { - freies Eintragen oder Einsprechen } \\
\text { - Foto-Upload und/oder Video-Upload } \\
\text { Hypothesen } \\
\text { - Überprüfung einer vorher definierten Hypothese } \\
\text { - Verwerfen oder Bestätigen der Hypothese } \\
\text { körperliche Untersuchung } \\
\text { - freies Eintragen oder Einsprechen } \\
\text { - Gliederung in } \\
\text { - Inspektion } \\
\text { - funktionelle Demo } \\
\text { - aktive Bewegung } \\
\text { - passive Bewegung } \\
\text { - Zusatztests } \\
\text { - Foto-Upload und/oder Video-Upload der } \\
\text { Bewegung } \\
\text { Probebehandlung } \\
\text { - freies Eintragen oder Einsprechen }\end{array}$ \\
\hline
\end{tabular}

Tab. 6 (Fortsetzung)

\begin{tabular}{|c|c|}
\hline \multirow[t]{2}{*}{ Kategorie } & Funktionalität \\
\hline & $\begin{array}{l}\text { visueller Messverlauf } \\
\text { - Eintragen oder Einsprechen eines Messwerts für } \\
\text { z. B. das Bewegungsausmaß eines Gelenks } \\
\text { (z. B. Abduktion SG rechts) } \\
\text { - zum aktuellen Zeitpunkt } \\
\text { - Verlaufskurve über die Zeit }\end{array}$ \\
\hline Therapieverlauf & $\begin{array}{l}\text { - automatisches Hinzufügen des Datums } \\
\text { - Jede nachträgliche Änderung der Verlaufsdoku- } \\
\text { mentation wird mit einem Zeitstempel und der } \\
\text { Benutzerkennung bzw. dem Therapeutenkürzel } \\
\text { versehen. }\end{array}$ \\
\hline Übersichtsseite & $\begin{array}{l}\text { - Zusammenfassung der Anamnese und des } \\
\text { Befundes } \\
\text { - Darstellung der positiven Testergebnisse } \\
\text { (inkl. VAS) } \\
\text { - Darstellung der Therapieziele } \\
\text { - Darstellung des bisherigen Behandlungsverlaufs } \\
\text { - Darstellung der bestätigten und verworfenen } \\
\text { Hypothesen }\end{array}$ \\
\hline Wiederbefund & $\begin{array}{l}\text { " Kontrolle der Therapieziele (erreicht, nicht } \\
\text { erreicht) } \\
\text { - Darstellung der positiven Testergebnisse }\end{array}$ \\
\hline Intervention & $\begin{array}{l}\text { - Hinzufügen und Entfernen einer Intervention } \\
\text { - Gliederung in } \\
\text { - Maßnahme } \\
\text { - Körpersegment } \\
\text { - Intensität } \\
\text { - Patientenreaktion } \\
\text { - Bemerkungen des Patienten } \\
\text { - freies Eintragen oder Einsprechen der } \\
\text { Unterpunkte } \\
\text { - Wiedervorlage von bereits durchgeführten } \\
\text { Interventionen }\end{array}$ \\
\hline $\begin{array}{l}\text { Hausaufgaben/ } \\
\text { Übungen }\end{array}$ & $\begin{array}{l}\text { - freies Eintragen oder Einsprechen } \\
\text { - Filmen der Übungsausführung } \\
\text { - Drucken und/oder Verschicken der Erklärungen } \\
\text { zur Übung }\end{array}$ \\
\hline $\begin{array}{l}\text { Abschluss- } \\
\text { befund }\end{array}$ & $\begin{array}{l}\text { - Gliederung in } \\
\text { - Entwicklung } \\
\text { - aktueller Stand } \\
\text { - Anzeigen eines visuellen Wertevergleichs } \\
\text { - Generieren des Abschlussbefundes mithilfe von } \\
\text { Textbausteinen }\end{array}$ \\
\hline Kommunikation & $\begin{array}{l}\text { Patient } \\
\text { - Versenden einer SMS, E-Mail o. ä. an Patienten } \\
\text { - Hausaufgabenliste und Erklärungen } \\
\text { Arzt } \\
\text { - Versenden des Arztberichts }\end{array}$ \\
\hline
\end{tabular}

Möglichkeit beinhalten sollte, die Inhaltsstruktur flexibel zu verwalten, und zum anderen, den Dokumentationsprozess zu unterstützen sowie Hilfestellungen im Clinical-Reasoning-Prozess aufzuzeigen. Zudem wird erwartet, dass durch eine intuitive Steuerung der Software auf mobilen Endgeräten eine Zeitersparnis erfolgt. Dabei könnte eine weitere Effizienzsteigerung durch 
einen digitalen Stammdaten- und Anamnesefragebogen eintreten, welcher von Patienten vor einer Behandlung ausgefüllt wird.

\section{Schlussfolgerungen}

Zusammenfassend zeigt die Befragung, dass eine softwarebasierte Dokumentation zum einen standardisierter als bisher durchgeführt werden sollte, um den Clinical-Reasoning-Prozess zu unterstützten. Zum anderen sollte eine entsprechende SoftwareAnwendung gleichzeitig eine gewisse Flexibilität bieten. Die gesammelten Anforderungen dienen der Entwicklung einer neuen benutzerorientierten mobilen Anwendung zur Effizienzsteigerung in der physiotherapeutischen Dokumentation.

\section{Interessenkonflikt}

Die Autorinnen/Autoren geben an, dass kein Interessenkonflikt besteht.

\section{Danksagung}

Die Autorinnen und Autoren danken allen Expertinnen und Experten für ihre Teilnahme sowie der Monitoring-Gruppe, bestehend aus Camilla Kapitza, Nadine Rensing, Sabine Bütterhoff, Christina Krone und Dirk Möller, für ihre Unterstützung bei den Pre-Tests.

\section{Literatur}

[1] Larenz K, Hrsg. Bürgerliches Gesetzbuch: mit Einführungsgesetz, Beurkundungsgesetz, AGB-Gesetz, Abzahlungsgesetz, Gesetz über den Widerruf von Haustürgeschäften, Wohnungseigentumsgesetz, Ehegesetz und Hausratsverordnung: Textausgabe mit ausführlichem Sachregister und einer Einführung. 30. Aufl. München: Deutscher TaschenbuchVerlag; 1987

[2] Krauskopf D, Hrsg. SGB V, Gesetzliche Krankenversicherung: mit Gesundheits-Reformgesetz (Auszug) Sozialgesetzbuch (SGB I), Allgemeiner Teil Sozialgesetzbuch (SGB IV), Gemeinsame Vorschriften für die Sozialversicherung: Textausgabe mit ausführlichem Sachregister und einer Einführung. 11., überarb. Aufl. München: Deutscher Taschenbuch-Verlag; 2002

[3] Kollak I, Bordiehn K. Was gehört in die Therapiedokumentation? In: Einfach dokumentieren. Berlin, Heidelberg: Springer; 2014: 5-22
[4] Rahmenverträge vdek und Berufsverbände. Im Internet (Stand 30.08.2019): www.vdek.com/vertragspartner/heilmittel/rahmenver trag/_jcr_content/par/download_14/file.res/RV_2013_UF.pdf

[5] Vergütungsvereinbarung 2018-2020 gemäß § 125 SGB V über Heilmittel der Physikalischen Therapie, 30-Aug-2019. Im Internet (Stand 24.08.2020): www.aok-gesundheitspartner.de/imperia/md/gpp/hh/heil berufe/vertraege/physiotherapie/20180501_hh_heil_physio_verguet_ 2018-2020_vtp.pdf

[6] Zalpour C. Lexikon Physiotherapie: mit 98 Tabellen. Berlin, Heidelberg: Springer; 2014

[7] Köster J, Kruse A, Kunze K. Clinical Reasoning: therapeutische Denkprozesse lernen. 2., überarb. und erw. Aufl. Stuttgart, New York: Thieme; 2015

[8] Michel-Verkerke MB. The Love-hate Relationship of Physical Therapists with Electronic Patient Records. Procedia Comp Science 2015; 64: 432441

[9] Krüger-Brand H. Dokumentation standardisieren. Bd. 109, Nr. 5/2012: A224. Im Internet (Stand 15.04.2020): www.aerzteblatt.de/archiv/tref fer?mode=s\&wo=1008\&typ=16\&aid=121117\&titel=dokumentation \& autor=Kr\%FCger\%2DBrand\%2C+Heike

[10] Jünger S, Payne SA, Brine J et al. Guidance on Conducting and REporting DElphi Studies (CREDES) in palliative care: Recommendations based on a methodological systematic review. Palliat Med 2017; 31: 684-706

[11] Gibson JM. Using the Delphi technique to identify the content and context of nurses' continuing professional development needs. J Clin Nurs 1998; 7: 451-459

[12] Powell C. The Delphi technique: myths and realities. J Adv Nurs 2003; 41 : 376-382

[13] Keeney S, Hasson F, McKenna H. Consulting the oracle: ten lessons from using the Delphi technique in nursing research. J Adv Nurs 2006; 53: 205-212

[14] Donohoe HM, Needham RD. Moving best practice forward: Delphi characteristics, advantages, potential problems, and solutions. Int J Tourism Res 2009; 11: 415-437

[15] Häder M. Delphi-Befragungen: ein Arbeitsbuch. 3. Aufl. Wiesbaden: Springer VS; 2014

[16] Cook C, Brismée JM, Pietrobon R et al. Development of a Quality Checklist Using Delphi Methods for Prescriptive Clinical Prediction Rules: The QUADCPR. Manipulative Physiol Ther 2010; 33: 29-41

[17] Kalra D. Electronic health record standards. Yearb Med Inform 2006: 136-144

[18] Vreeman DJ, Taggard SL, Rhine MD et al. Evidence for electronic health record systems in physical therapy. Phys Ther 2006; 86: 434-446, discussion 446-449 